\title{
An Ulceronecrotic Foot Lesion in a Patient with Essential Thrombocythemia: Successful Treatment with Hydroxyurea
}

\author{
Tokue Kato Seiji Kawana \\ Department of Dermatology, Nippon Medical School, Tokyo, Japan
}

\section{Key Words}

Essential thrombocythemia $\cdot$ Hydroxyurea $\cdot$ Skin ulcer $\cdot$ Livedo

\begin{abstract}
The patient was a 47-year-old woman with a painful ulcer that had appeared on the right 5th toe two weeks before she visited our hospital. Histopathological examination showed that thrombi were present in small blood vessels in the dermis and pancytosis was detected in a blood test, suggesting polycythemia-associated ulceration of the toe. Essential thrombocythemia was diagnosed based on bone marrow puncture and chromosomal test findings. Platelet count and the ulcer were improved by oral hydroxyurea.
\end{abstract}

\section{Introduction}

Chronic myeloproliferative diseases include essential thrombocythemia (ET), polycythemia vera, chronic myelocytic leukemia and myelofibrosis, and cause hemorrhagic and thrombotic symptoms in many organs of the body [1]. Purpura, subcutaneous hemorrhage, ulcer, acral gangrene, livedo symptoms, and erythromelalgia can occur in the skin [2]. We encountered a patient in whom an ETassociated ulcer of the toe was markedly improved by treatment with hydroxyurea.

\section{Case Report}

The patient was a 47-year-old woman in whom an ulcer had developed on the toe two weeks before she visited the Outpatient Clinic of our department because of pain. Polycythemia and hypertension had been found during a health check-up 4 years earlier, but had been left untreated. A purple-reddish macula accompanied by coldness and pain had appeared on the toe tip 3 years before and the region had repeatedly swelled for 2 years. On the first examination, a swelled, cyanotic 
discoloration of the skin was present on the right 5 th toe. An ulcer $5 \mathrm{~mm}$ in diameter, covered with a crust and accompanied by pain and numbness was noted at the toe tip ( $\underline{\text { fig. } \mathbf{1}} \mathbf{a}, \mathrm{b}$ ). Livedo was also present on the back and side of the right foot. Pulsation of the dorsal artery of the foot was good on both sides and intermittent claudication was absent. Histopathological examination of the ulcerated skin showed that the epidermis was partially defective, platelet thrombi were present in dilated small blood vessels in the upper to middle dermis, and inflammatory cells had infiltrated around the small vessels (fig. 2a). The inner wall was destroyed in some blood vessels and the structure was unclear with complete obstruction of the lumen (fig. 2 b). No cholesterol crystals were present in the thrombotic region.

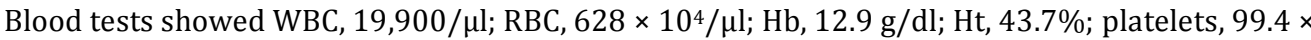
$10^{4} / \mu \mathrm{l}$; IgG, 1,458 mg/dl; IgA, $418 \mathrm{mg} / \mathrm{dl} ; \mathrm{IgM}, 135 \mathrm{mg} / \mathrm{dl}$; ferritin, $6.3 \mathrm{ng} / \mathrm{ml}$; PR3-ANCA, negative; MPO-ANCA, negative; anticardiolipin antibody, negative; cryoglobulin, negative; and vitamin B12, 812 $\mathrm{ng} / \mathrm{ml}$ (normal value: 200-800). In a bone marrow test, the number of irregular-sized megakaryocytes with atypical nuclei was markedly increased, and the numbers of granulocyte system cells and erythrocyte precursors were also increased, but no myelofibrosis was noted. Philadelphia chromosome was negative and no other chromosomal aberrations were detected. Abdominal echography detected a $67 \times 44$-mm splenomegaly, but there were no findings suggesting infection, inflammatory disease, or malignant tumor. Based on these findings, ET and an accompanying skin ulcer were diagnosed. Administration of oral hydroxyurea at $500 \mathrm{mg} /$ day was initiated and the patient's platelet count was reduced to $42 \times 10^{4} / \mu \mathrm{l}$ and WBC to $9,700 / \mu \mathrm{l}$ after several months. The ulcer on the right 5 th toe was mostly epithelialized about one month after initiation of hydroxyurea, and only mild livedo and pigmentation were present thereafter.

\section{Discussion}

ET is one of several chronic myeloproliferative diseases and has a pathology believed to involve hyperplasia of platelets without normal function due to abnormal autonomous proliferation of megakaryocyte-lineage cells in the bone marrow. ET is diagnosed based on a platelet count of $60 \times 10^{4} / \mu \mathrm{l}$ or higher and $\mathrm{Ht}$ of $40 \%$ or lower, normal RBCs, normal serum ferritin, a Philadelphia chromosome- or bcr/abl fusion gene-negative status, the exclusion of chronic myelocytic leukemia, the absence of marked myelofibrosis, and the exclusion of reactive thrombocythemia [1]. The incidence is high in persons aged 60 years and older, and the condition may be accompanied by deep or superficial venous thrombosis, nasal hemorrhage, intracranial hemorrhage, transient ischemic attack, and cerebral and myocardial infarction, although many cases are asymptomatic [2]. Regarding skin symptoms, purpura is most frequent, followed by erythromelalgia, skin ulcer, and livedo reticularis [2].

Skin symptoms in ET such as ulcer and gangrene are thought to develop due to an increased production of platelet-activating substances such as thromboxane $\mathrm{A}_{2}$ and prostaglandins $\mathrm{G}_{2}$ and $\mathrm{H}_{2}$. This process is induced by an increased adhesion, release and aggregation due to an increased platelet count, elevated sensitivity of platelets to these substances, decreased production of prostacyclin, which is a platelet-function inhibitor, and increased platelet aggregation due to decreased sensitivity of platelets to prostaglandins $\mathrm{I}_{2}$ and $\mathrm{D}_{2}[1-4]$.

To our knowledge, 25 patients with ET-associated skin ulcer/necrosis of the toe have been reported since 1970 [2, 5-8]. Two patients were treated with hydroxyurea and the ulcer markedly improved [7, 8], whereas 7 patients were treated with antiplatelet drugs such as aspirin, but gangrene progressed and amputation of the toe was required. Hydroxyurea is a metabolic antagonist that inhibits DNA synthesis, and it is frequently used in the treatment of myeloproliferative diseases such as chronic 
myelocytic leukemia. Hydroxyurea has been used to treat ET in many cases since the 1990s [1, 7, 8], with the goal of reducing the platelet count [9-11]. The patient in the current case was treated with hydroxyurea from the early phase, and the platelet count decreased and skin symptoms were improved. Hydroxyurea treatment at $500 \mathrm{mg} /$ day is ongoing and her platelet count has stabilized at about $50-70 \times 10^{4} / \mu \mathrm{l}$ without recurrence of skin symptoms. It sometimes use treated for skin ulcer with antiplatelet drugs such as aspirin or cilostazol, cyclophosphamide for reducing the number of platelet [2].

Our case suggests that ET should be kept in mind as a possible cause of refractory ulcer in the crura and toes, even though the condition is rare. In such cases, early diagnosis and rapid treatment may prevent aggravation, and hydroxyurea is the treatment of choice [11-13].
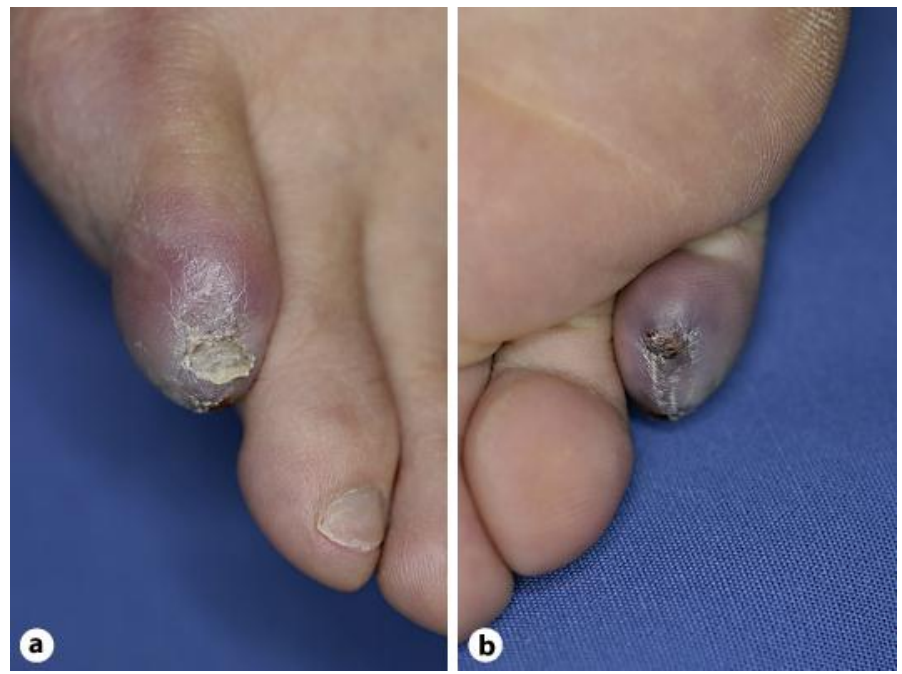

Fig. 1. Skin symptoms. The right 5 th toe was swollen and had a purple-reddish color (a). A crusted ulcer was present on the ventral side of the toe (b). 

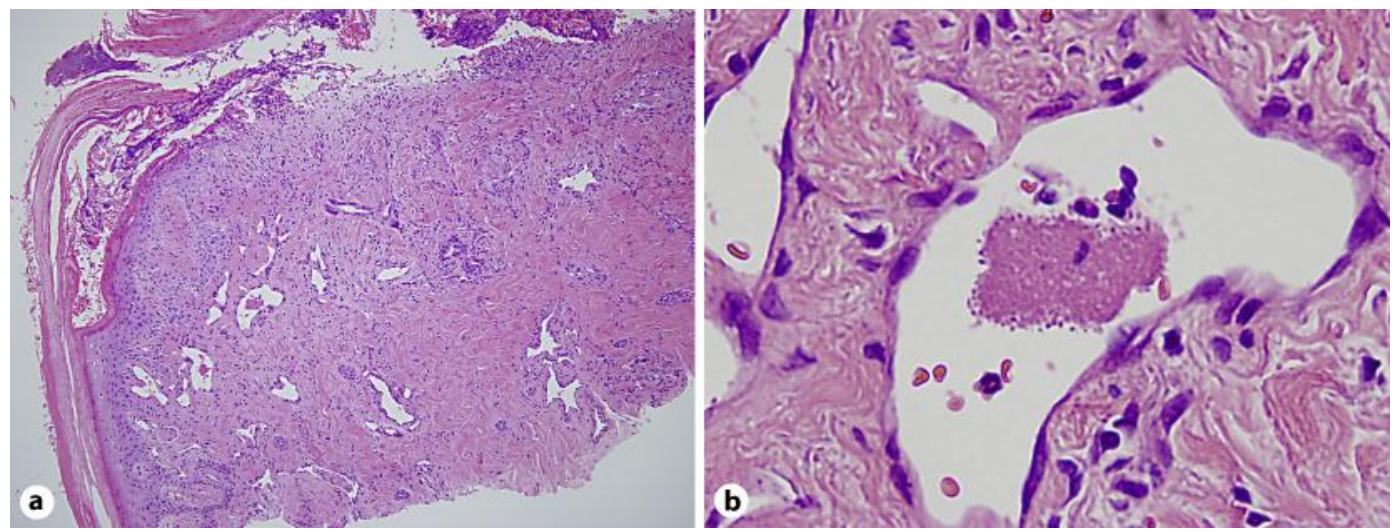

Fig. 2. Histopathological findings (hematoxylin and eosin staining). Hyperkeratosis, parakeratosis, and a partial defect were noted in the epidermis. Inflammatory cells infiltrated around dermal blood vessels (a) $(\times 40)$, and the blood vessels were partially dilated and contained platelet thrombi (b) $(\times 400)$.

\section{References}

1 Murphy S, Iland H, Rosenthal D, Laszio J: Essential thrombocythemia: an interim report from the Polycythemia Vera Study Group. Semin Hematol 1986;23:177-182.

$\checkmark 2$ Itin PH, Winkelmann RK: Cutaneous manifestations in patients with essential thrombocythemia. J Am Acad Dermatol 1991;24:59-63.

3 Michiels JJ, van Joost T: Erythromelalgia and thrombocythemia: a causal relation. J Am Acad Dermatol 1990;22:107-111.

4 Schafer AI: Bleeding and thrombosis in the myeloproliferative disorders. Blood 1984;64:1-12.

5 Gillespie G: Peripheral gangrene as the presentation of myeloproliferative disorders. Br J Surg 1973;60:377-380.

6 Preston FE, Emmanuel IG, Winfield DA, Malia RG: Essential thrombocythemia and peripheral gangrene. Br Med J 1974;3:548-552.

7 Velasco J A, Santos JC, Blavo J, Santana J: Ulceronecrotic lesions in a patient with essential thrombocythemia. Clin Experimental Dermatol 1991;16:53-54.

8 Suarez Conde I, Peterio C, Peterio Ferreiros MM, Toribio J: Cutaneous vasculitis revealing essential thrombocythemia. Dermatology 2002;205:75-76.

-9 Lofvenberg E, Wahlin A: Management of polycythemia vera, essential thrombocythaemia and myelofibrosis with hydroxyurea. Eur J Haematol 1988;41:375-381.

10 Griesshammer M, Bangerter M, Sauer T, Wennauer R, Bergmann L, Heimpel H: Aetiology and clinical significance of thrombocytosis: analysis of 732 patients with an elevated platelet count. J Intern Med 1999;245:295-300.

11 Schafer AI: Molecular basis of the diagnosis and treatment of polycythemia vera and essential thrombocythemia. Blood 2006;107:4214-4222.

12 Cortelazzo S, Finazzi G, Ruggeri M, et al: Hydroxyurea for patients with essential thrombocythemia and a high risk of thrombosis. N Engl J Med 1995;332:1132-1136.

13 Finazzi G, Ruggeri M, Rodeghiero F, Barbui T: Efficacy and safety of long-term use of hydroxyurea in young patients with essential thrombocythemia and a high risk of thrombosis. Blood 2003;101:3749. 